\title{
How Helpful Is aEEG? Context and User Experience Matter
}

\author{
Amanda G. Sandoval Karamian, MD ${ }^{1}$ Courtney J. Wusthoff, MD, MS ${ }^{1,2}$ \\ ${ }^{1}$ Division of Child Neurology, Children's Hospital of Philadelphia, \\ Philadelphia, Pennsylvania \\ 2 Division of Child Neurology and Division of Pediatrics-Neonatal and \\ Developmental Medicine, Stanford University, Palo Alto, California \\ Address for correspondence Amanda G. Sandoval Karamian, MD, \\ 3501 Civic Center Boulevard, Office 1200.12, Philadelphia, MA 19104 \\ (e-mail: sandovalka@email.chop.edu).
}

Am J Perinatol 2022;39:1132-1137.

\begin{abstract}
Keywords

- amplitude-integrated EEG

- neonatal neurology

- neonatal seizures

- brain monitoring

Objective The aim of the study is to model amplitude-integrated electroencephalography (aEEG) utility to diagnose seizures in common clinical scenarios.

Study Design Using reported neonatal seizure prevalence and aEEG sensitivities and specificities, likelihood ratios (LRs) and post-test probabilities were calculated to quantify aEEG utility to diagnose seizures in three typical clinical scenarios.

Results Prevalence data supported pretest probabilities for neonatal seizures of 0.4 in neonatal hypoxic ischemic encephalopathy (HIE), 0.27 in bacterial meningitis, and 0.05 in extreme prematurity. Reported sensitivity of $85 \%$ and specificity of $90 \%$ for seizures with expert aEEG interpretation yielded a positive likelihood ratio (LR+) of 8.7 and a negative likelihood ratio (LR-) of 0.17 . Reported sensitivity of $65 \%$ and specificity of $70 \%$ with intermediate interpretation yielded LR+ 2.17 and LR- 0.5 . Reported sensitivity of $40 \%$ and sensitivity of $50 \%$ with inexperienced interpretation gave $L R+0.8$ and $L R-1.2$. These translate the ability to move pretest to post-test probability highly dependent on user expertise. For HIE, a pretest probability of seizure of 0.4 moves to a post-test probability of 0.85 when aEEG is positive for seizures by expert interpretation, and down to 0.1 when aEEG is negative. In contrast, no useful information was gained between pretest and post-test probability by aEEG interpreted as negative or positive for seizure at the inexperienced user level. Similarly, in the models of meningitis or extreme prematurity, incremental information gained from aEEG ranged widely based on interpreter experience.

Conclusion aEEG is most useful to screen for neonatal seizures when used in conditions with high seizure prevalence, and when interpretation has a sensitivity and specificity as reported for expert users. In contrast, aEEG can become negligible in providing meaningful clinical information when applied in conditions having lower seizure prevalence or when interpretation has low accuracy. Appropriate patient selection and high quality interpretation are essential for $\mathrm{aEEG}$ utility in neonatal seizure detection.
\end{abstract}

\section{Key Points}

- aEEG utility for neonatal seizure screening relies on patient selection and quality interpretation.

- Utility of aEEG is highest with high seizure prevalence and expert interpretation.

- Utility of aEEG can be negligible with lower seizure prevalence or low accuracy interpretation.

received

June 4, 2020

accepted after revision

November 2, 2020

published online

December 15, 2020
DOI https://doi.org/

$10.1055 / \mathrm{s}-0040-1721711$. ISSN 0735-1631.

\footnotetext{
(C) 2020. The Author(s).

This is an open access article published by Thieme under the terms of the Creative Commons Attribution-NonDerivative-NonCommercial-License, permitting copying and reproduction so long as the original work is given appropriate credit. Contents may not be used for commercial purposes, or adapted, remixed, transformed or built upon. (https://creativecommons.org/ licenses/by-nc-nd/4.0/)

Thieme Medical Publishers, Inc., 333 Seventh Avenue, 18th Floor, New York, NY 10001, USA
} 
Amplitude-integrated electroencephalography (aEEG) is an increasingly popular tool to screen for neonatal seizures. aEEG uses a limited number of channels to record raw electroencephalography (EEG) data which is filtered, processed, and displayed on a semilogarithmic amplitude and time-compressed scale. ${ }^{1}$ Seizures appear as abrupt increases in the voltage on the compressed tracing. ${ }^{1,2}$ There are several challenges in the interpretation of aEEG. Due to the time compression of a standard aEEG display, brief neonatal seizures may be missed, as a seizure lasting 90 seconds would only appear for $1.4 \mathrm{~mm}$ on the screen; seizures lasting less than 30 seconds may not be detected. ${ }^{1,2}$ As aEEG filters out frequencies slower than $2 \mathrm{~Hz}$, neonatal seizures which are of very low frequency may be filtered out of the tracing. ${ }^{1}$ Due to the limited electrode array focused on the central/parietal regions, focal neonatal seizures which are outside of these regions may not be detected by aEEG. ${ }^{3}$ Additionally, artifacts are very common in aEEG and it can be difficult to distinguish seizure from artifacts such as patting, bedside nursing care, electrocardiographic artifact, or electrode malfunction. ${ }^{1}$

While expert aEEG interpretation has been shown to have good sensitivity and specificity for seizures, typical use may have lower accuracy. The utility of any screening test depends upon how well the test result refines an estimate that a patient has a given condition, moving the pretest probability to a more accurate post-test probability. The potential utility of aEEG to screen for seizures is dependent on the risk of seizures in the patient population undergoing aEEG (pretest probability) and accuracy of the aEEG when interpreted by providers of varying skill levels (test characteristics). For a neonate at risk for seizures, the pretest probability may be estimated based on published evidence regarding the prevalence of seizures in a specific population. A likelihood ratio (LR) incorporates sensitivity and specificity to assess test utility. Reported sensitivities and specificities for either diagnosing or ruling out seizures range widely, largely dependent on user skill in aEEG interpretation. This produces varying evidence-based LRs for aEEG in different described contexts. To demonstrate the importance of seizure prevalence and expertise in aEEG interpretation, we applied statistical principles to model utility of aEEG to move the pretest probability of seizures to a more accurate posttest probability across three common clinical scenarios.

\section{Materials and Methods}

The prevalence, sensitivity, and specificity of aEEG were derived from reported values in the literature. The prevalence of seizures in three clinical conditions affecting neonates (hypoxic ischemic encephalopathy [HIE] in term neonates $\geq 37$ weeks GA, ${ }^{4}$ bacterial meningitis in term and late preterm neonates $\geq 33$ weeks $G A,{ }^{5,6}$ and prematurity in neonates $<30$ weeks $\mathrm{GA}^{7-9}$ ) were used to derive the pretest probability of seizures for scenarios based on each condition. The sensitivity and specificity of aEEG for seizure detection were derived from the literature for three models of aEEG interpretation based on experience level: ideal/expert inter- pretation, ${ }^{10-12}$ intermediate interpretation, ${ }^{10,13}$ and the lowest reported values with inexperienced users. ${ }^{10,14,15}$ Expert interpretation of aEEG was performed by senior neonatologists with experience interpreting aEEG and epileptologists with experience reading aEEG. ${ }^{10-12}$ Intermediate interpretation was performed by epileptologists with experience reading conventional EEG and neonatology fellows. ${ }^{10,13,15}$ Inexperienced interpretation was performed by medical students and neonatologists with no prior experience interpreting aEEG. ${ }^{10,14,15}$ The utility of aEEG for seizure screening was calculated via post-test probabilities from LRs.

$$
\begin{gathered}
\text { pretest odds }=\frac{\text { pretest probability }}{1-\text { pretest probability }} \\
\text { pretest odds }=\frac{\text { pretest probability }}{1-\text { pretest probability }} \\
\text { post }- \text { test probability }=\frac{\text { post }- \text { test odds }}{1+\text { post }- \text { test odds }} \\
\text { post }- \text { test odds }=\text { pretest odds X L R } \\
L R+=\frac{\text { sensitivity }}{1-\text { specificity }} \quad \text { LR }-=\frac{1-\text { sensitivity }}{\text { specificity }}
\end{gathered}
$$

\section{Results}

The first scenario considered an example of HIE, reported to confer a relatively high risk of neonatal seizures. - Table 1 shows the reported sensitivity and specificity from the literature applied to model differences in aEEG interpretation between the expert, intermediate, and inexperienced levels. The reported prevalence of seizures in hypoxic-ischemic encephalopathy is approximately $40 \%{ }^{4}$ The best reported sensitivity for aEEG in seizure detection is $85 \%$, specificity is $90 \%{ }^{10-12}$ Applying these reported values to model expert interpretation yields a positive likelihood ratio $(\mathrm{LR}+)$ of 8.5 and a negative likelihood ratio (LR-) of 0.17 for this test. Therefore, under this model a neonate with HIE starts with a general pretest probability of 0.4 for seizures (based on $40 \%$ prevalence in the literature), in the absence of any information from aEEG. If expert interpretation finds

Table 1 Models of aEEG interpretation based on reported sensitivity and specificity for expert level users, intermediate level interpretation, and the lowest reported values

\begin{tabular}{|llll|}
\hline & $\begin{array}{l}\text { Expert } \\
\text { interp- } \\
\text { retation }\end{array}$ & $\begin{array}{l}\text { Intermediate } \\
\text { interpretation }\end{array}$ & $\begin{array}{l}\text { Inexperienced } \\
\text { interpretation }\end{array}$ \\
$\begin{array}{l}\text { Sensitivity } \\
\text { Specificity }\end{array}$ & $85 \%$ & $65 \%$ & $40 \%$ \\
$\begin{array}{l}\text { Positive } \\
\text { likelihood } \\
\text { ratio }\end{array}$ & 8.5 & 2.17 & $50 \%$ \\
$\begin{array}{l}\text { Negative } \\
\text { likelihood } \\
\text { ratio }\end{array}$ & 0.17 & 0.5 & 0.80 \\
\hline
\end{tabular}

Abbreviation: aEEG, amplitude-integrated electroencephalography. 


\section{Hypoxic Ischemic Encephalopathy}

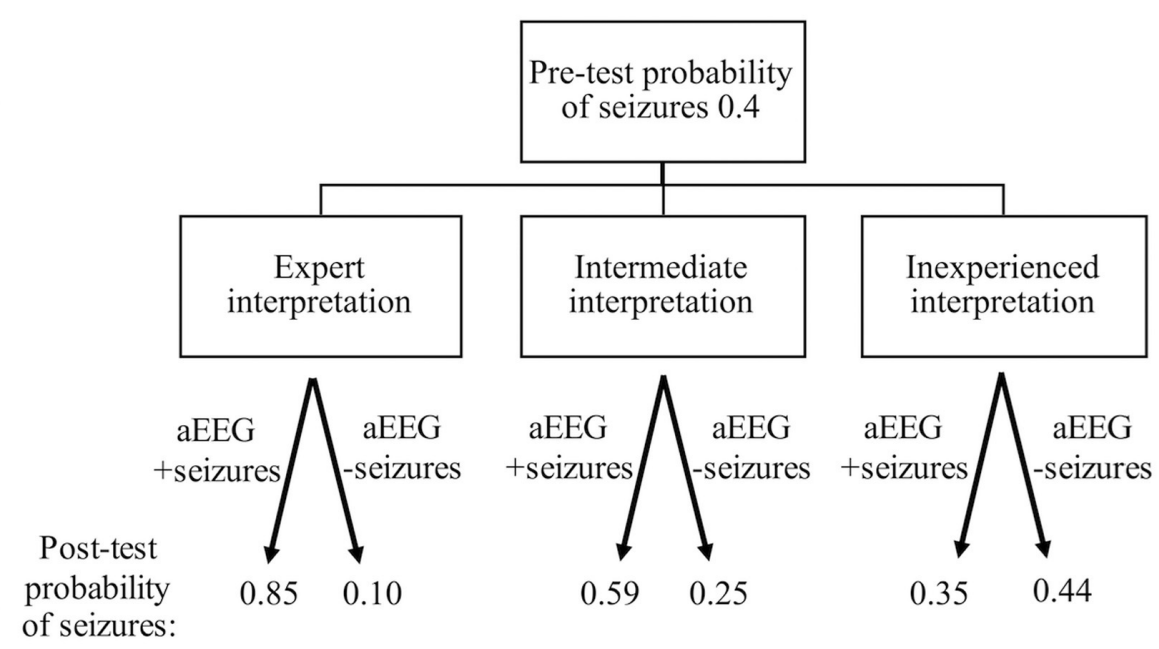

Fig. 1 Model of aEEG interpretation for users with varied experience levels in neonates with HIE, with reported seizure prevalence of $40 \%$. aEEG, amplitude-integrated electroencephalography; HIE, hypoxic ischemic encephalopathy.

seizures on aEEG, an individual patient then has a post-test probability of 0.85 of true seizures, reflecting a considerable change in the estimated seizure risk for that neonate ( - Fig. 1 ). At the same time, a negative aEEG by expert interpretation gives a post-test probability of 0.1 for seizures, which is meaningfully different from the pretest estimate of 0.4. The usefulness of the test changes if there is lower accuracy in aEEG interpretation. Using moderate reported values of sensitivity (65\%) and specificity $(70 \%)^{10,13}$ to model intermediate interpretation skill, aEEG has LR+ of 2.17 and LR- 0.5. This means that for a neonate with HIE, again starting with a pretest probability of 0.4 for seizures, an aEEG positive for seizures by intermediate interpretation translates to a post-test probability of only 0.59 for that patient having true seizures. Likewise, a neonate with HIE having an aEEG negative for seizures by intermediate interpretation still has a post-test probability of 0.25 that true seizures are present. Finally, the least accurate, inexperienced interpretation was modeled using the lowest reported test characteristics among users with limited aEEG accuracy (sensitivity $=40 \%$, specificity $=50 \%$ ), ${ }^{10,14,15}$ with $L R+0.8$ and LR-1.2. This again was applied to the neonate with HIE who is known to have a risk of seizures of 0.4 without aEEG information-if the least accurate, inexperienced user finds that patient's aEEG positive for seizures, the post-test probability of true seizures is only 0.35 . Similarly, if the inexperienced user finds the aEEG negative for seizures, there remains a 0.44 post-test probability of true seizures. Under the poor test characteristics for inexperienced aEEG interpretation, based on the least accurate sensitivity and specificity previously reported, the test paradoxically makes the estimate of seizure risk less accurate than it was prior to the test results.

Another clinical scenario with risk of seizures is neonatal bacterial meningitis. The reported prevalence of seizures in neonates with bacterial meningitis ranges from 20 to $34 \%$, $^{5,6}$
Based on this, a prevalence of $27 \%$ can be used to model aEEG utility based on experience level, using the same test characteristics detailed above ( - Fig. 2). From a pretest probability of 0.27 for seizures in neonatal bacterial meningitis without aEEG information available, an aEEG positive for seizures by expert interpretation leads to a more accurate post-test probability of 0.76 for true seizures. Conversely, if aEEG is negative for seizures by expert interpretation, the post-test probability of true seizures is only 0.06 . Using intermediate interpretation, an aEEG positive for seizures increases the post-test probability of true seizures to 0.45 . While this is a meaningfully higher probability of seizures than was estimated before the test, it is notable that an aEEG positive for seizures in this situation still only means there is a $45 \%$ chance an individual patient truly has seizures. Conversely, an aEEG negative for seizures by intermediate interpretation creates a post-test probability of 0.16 for true seizures. Finally, in the model of lower reported sensitivity and specificity as above, the post-test probability of true seizures after positive aEEG is only 0.23 and is 0.31 even after negative aEEG.

Finally, we can model the use of aEEG for neonates with lower risk for seizures (low pretest probability), using the example of reported prevalence of EEG confirmed seizures in extremely and very preterm ( $<30$ weeks gestational age) neonates of $5 \%{ }^{7-9}$ Because the post-test probability of a condition depends both on the test characteristics and the pretest prevalence of the condition, aEEG used to screen for seizures in patients at lower risk for seizures yields lower overall post-test probabilities for true seizures, even if aEEG is positive for seizures. Expert interpretation (sensitivity $85 \%$, specificity $90 \%$ ) was applied to the scenario of the extremely preterm neonate using the LR and prevalence data above ( - Fig. 3). Starting with a pretest probability of 0.05 for seizure, an aEEG positive for seizure by expert interpretation results in a post-test probability of 0.31 for 


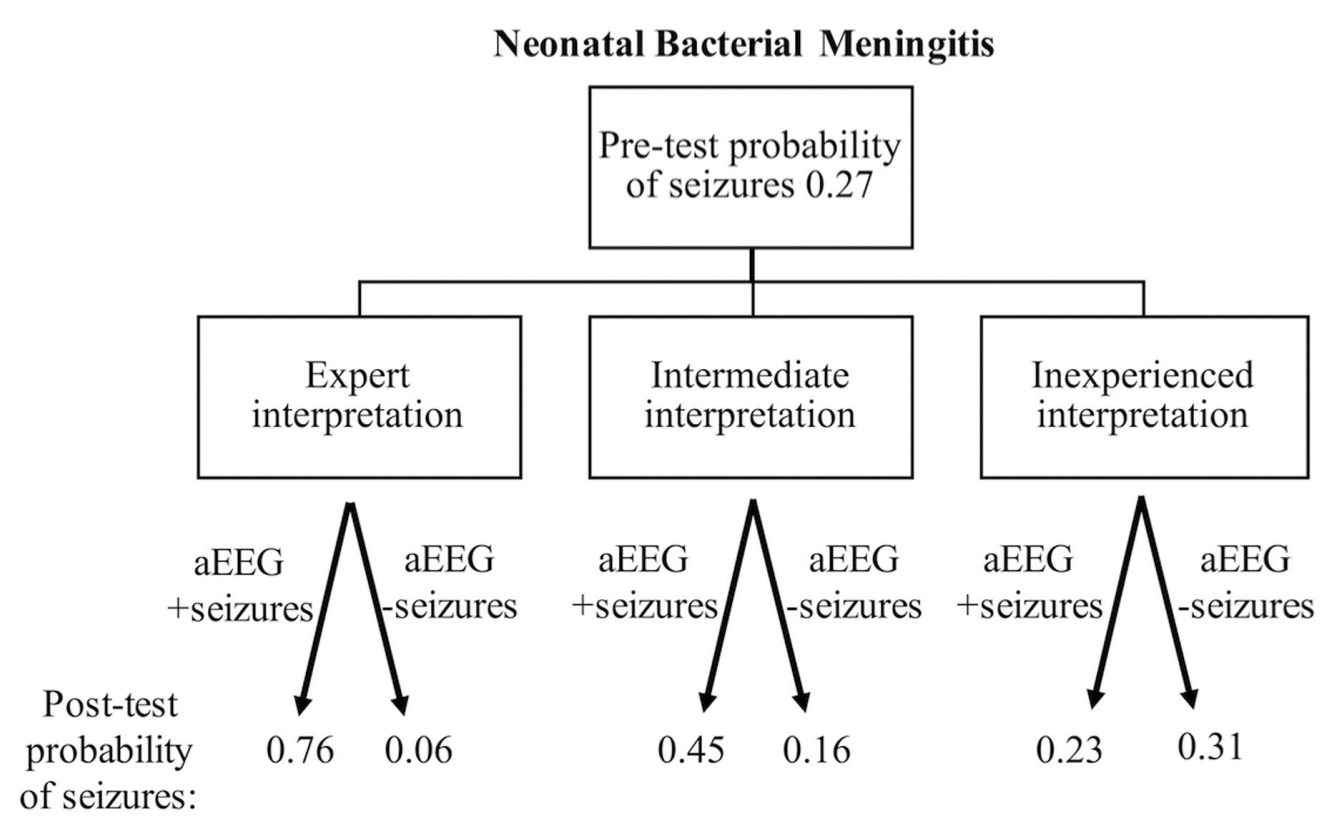

Fig. 2 Model of aEEG interpretation for users with varied experience levels in neonates with bacterial meningitis, with reported seizure prevalence of $27 \%$. aEEG, amplitude-integrated electroencephalography.

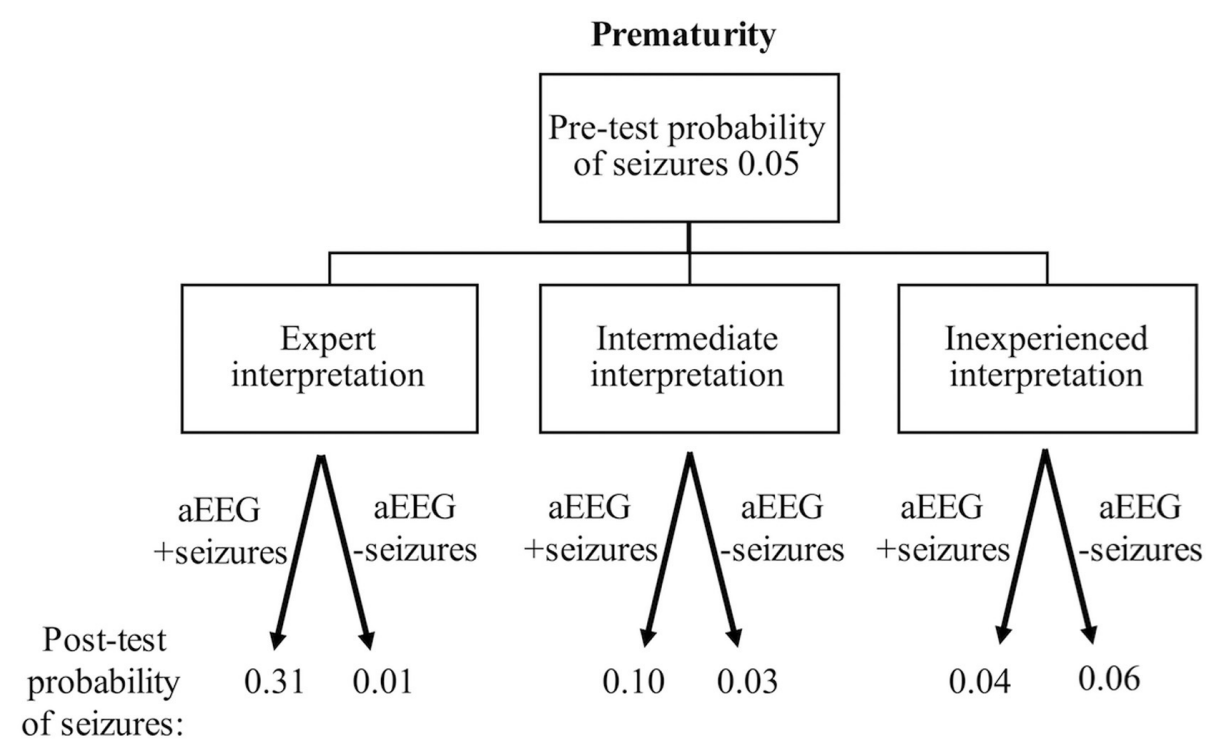

Fig. 3 Model of aEEG interpretation for users with varied experience levels in premature neonates with gestational age $<30$ weeks, with reported seizure prevalence of $5 \%$. aEEG, amplitude-integrated electroencephalography.

true seizures, meaning the patient is more likely to have a false positive aEEG than true seizures. The post-test probability for true seizures is 0.01 after negative aEEG by expert interpretation. Intermediate interpretation (sensitivity $=65 \%$, specificity $=70 \%$ ) carries even lower utility. This gives post-test probabilities of 0.1 for true seizures after positive aEEG, and 0.03 of seizures after negative aEEG. For the least accurate users (sensitivity $=40 \%$, specificity $=50 \%$ ), posttest probabilities of 0.04 of seizures after positive aEEG and 0.06 of seizures after negative aEEG reflect no gain of any useful information.

\section{Discussion}

With sufficiently high pretest probability of seizures and expert level accuracy in interpretation, corresponding to high sensitivity and specificity, aEEG is a useful screening tool for the detection of neonatal seizures. In neonates with HIE and expert level interpretation, post-test probabilities of 0.85 for true seizures after positive aEEG and 0.1 for seizures after negative aEEG make this a helpful and informative tool. However, aEEG was moderately useful in the setting of HIE (high pretest probability) with a nonexpert, intermediate 
level of interpretation. Worrisomely, when the lowest reported sensitivity and specificity was applied to the scenario of HIE, there was no meaningful information gained from the test, with post-test probabilities of 0.35 of having seizures after a positive aEEG, and 0.44 of seizures after a negative aEEG.

As the pretest probability of seizures gets lower for different clinical scenarios, the utility of aEEG decreases as well. In neonates with bacterial meningitis with a seizure prevalence of approximately $27 \%$, aEEG remained a useful tool under conditions of expert interpretation, with post-test probabilities of 0.76 for true seizures after positive aEEG and 0.06 of seizures after negative aEEG. However, with intermediate level interpretation, the post-test probability of true seizures was below 0.5 after positive aEEG. This suggests that while a positive aEEG might identify those neonates with a relatively higher risk of seizures to undergo more definitive testing, caution should be used before initiating seizure treatment based on a positive aEEG in such a scenario. With the lowest reported sensitivity and specificity, there was again no meaningful information added from the test.

The scenario of the extremely preterm neonate illustrates a case that is very common, and also reflects the need for caution before acting on aEEG results in a neonate at low risk for seizures. Extremely preterm neonates have been reported to have an overall seizure prevalence of $5 \%$ when the gold standard of full array continuous EEG monitoring is applied prospectively. ${ }^{7}$ Because this is a relatively low pretest probability of seizures, even with the most expert level of aEEG interpretation, a positive test is not necessarily diagnostic. In our model, even with expert interpretation, the post-test probability of true seizures after positive aEEG was only 0.31 . Again, while this may be high enough to justify more intensive diagnostics or monitoring, it may not be appropriate to initiate treatment based on this post-test probability alone.

This study has important limitations. These theoretical models, while based on evidence reported in the literature, cannot capture the nuances of every potential clinical scenario. We do not mean to claim that the post-test probabilities calculated here are accurate for actual individual patients. Rather, we chose these cases to illustrate examples of where aEEG could be most useful and when it might have lower utility. The pretest probabilities used are based on estimates of seizure prevalence for the selected conditions reported in the literature from population-based studies of large cohorts of neonates. The clinical scenarios outlined were selected to offer conditions commonly encountered in the neonatal intensive care unit with differing seizure prevalence to highlight the impact this has on test utility. Our estimates of the test characteristics sensitivity and specificity are based on those published across multiple studies of aEEG accuracy. Some of these studies used older equipment or methods of review, such as single-channel aEEG, that are less relevant to current practice at many centers. The estimates here for "expert," "intermediate," and "inexperienced" accuracy are only estimates; individual users may fall in between these categories or vary in accuracy in different situations. At the same time, these examples clearly illustrate the impact of varying skill in interpretation-with lower accuracy, aEEG gives much less useful information. This suggests that those using aEEG for seizure detection would benefit from having insight into their own skill level, thus recognizing their approximate sensitivity and specificity in interpretation. It also creates an opportunity to improve aEEG utility as increased experience and training to improve interpretation skill will improve test characteristics for a given user. Finally, we do not offer primary evidence from actual patient populations regarding how aEEG may improve care or change outcomes. This work uses only statistical approaches to illustrate examples of how patient selection and skill in interpretation impact aEEG utility, and should not be considered as definitive recommendations for which patients should or should not be monitored with aEEG.

\section{Conclusion}

aEEG is very useful when appropriately used to screen for neonatal seizures. However, under some circumstances it can be much less helpful and even negligible. Providers using aEEG must recognize the importance of patient selection for aEEG based on evidence of sufficiently high pretest probability. Similarly, insight into skill level of aEEG interpretations at one's own center can inform test utility in a specific context. Continuing training and education in aEEG interpretation will allow users to improve the test characteristics at their sites, while further research regarding prevalence of neonatal seizures in different populations is needed to inform pretest probability estimates.

\section{Conflict of Interest}

C.J.W. is supported by NIH/NINDS K02NS102598. The other author declares no conflict of interest.

\section{References}

1 Glass HC, Wusthoff CJ, Shellhaas RA. Amplitude-integrated electro-encephalography: the child neurologist's perspective. J Child Neurol 2013;28(10):1342-1350

2 Hellström-Westas L. Amplitude-integrated electroencephalography for seizure detection in newborn infants. Semin Fetal Neonatal Med 2018;23(03):175-182

3 Wusthoff CJ, Shellhaas RA, Clancy RR. Limitations of singlechannel EEG on the forehead for neonatal seizure detection. J Perinatol 2009;29(03):237-242

4 Glass HC, Shellhaas RA, Wusthoff CJ, et al. Neonatal Seizure Registry Study Group. Contemporary profile of seizures in neonates: a prospective cohort study. J Pediatr 2016; 174:98-103.e1

5 Gaschignard J, Levy C, Romain O, et al. Neonatal bacterial meningitis: 444 cases in 7 years. Pediatr Infect Dis J 2011;30(03): 212-217

6 Baud O, Aujard Y. Neonatal bacterial meningitis. Handb Clin Neurol 2013;112:1109-1113

7 Lloyd RO, O'Toole JM, Pavlidis E, Filan PM, Boylan GB. Electrographic seizures during the early postnatal period in preterm infants. J Pediatr 2017;187:18-25.e2

8 Scher MS, Aso K, Beggarly ME, Hamid MY, Steppe DA, Painter MJ. Electrographic seizures in preterm and full-term neonates: clinical correlates, associated brain lesions, and risk for neurologic sequelae. Pediatrics 1993;91(01):128-134 
9 Le Bihannic A, Beauvais K, Busnel A, de Barace C, Furby A. Prognostic value of EEG in very premature newborns. Arch Dis Child Fetal Neonatal Ed 2012;97(02):F106-F109

10 Frenkel N, Friger M, Meledin I, et al. Neonatal seizure recognition -comparative study of continuous-amplitude integrated EEG versus short conventional EEG recordings. Clin Neurophysiol 2011;122(06):1091-1097

11 Lawrence R, Mathur A, Nguyen The Tich S, Zempel J, Inder T. A pilot study of continuous limited-channel aEEG in term infants with encephalopathy. J Pediatr 2009;154(06):835-41.e1

12 Shah DK, Mackay MT, Lavery S, et al. Accuracy of bedside electroencephalographic monitoring in comparison with simul- taneous continuous conventional electroencephalography for seizure detection in term infants. Pediatrics 2008;121(06): 1146-1154

13 Zhang L, Zhou Y-X, Chang L-W, Luo X-P. Diagnostic value of amplitude-integrated electroencephalogram in neonatal seizures. Neurosci Bull 2011;27(04):251-257

14 Rennie JM, Chorley G, Boylan GB, Pressler R, Nguyen Y, Hooper R. Nonexpert use of the cerebral function monitor for neonatal seizure detection. Arch Dis Child Fetal Neonatal Ed 2004;89(01):F37-F40

15 Shellhaas RA, Soaita AI, Clancy RR. Sensitivity of amplitudeintegrated electroencephalography for neonatal seizure detection. Pediatrics 2007;120(04):770-777 